\title{
Mesenchymal stem cells in osteoarticular pediatric diseases: an update
}

\author{
German A. Norambuena', Maroun Khoury' and Christian Jorgensen²
}

Cellular therapy has gained an increasing popularity in recent years. Mesenchymal stem cells (MSCs) have the potential to differentiate into bone, cartilage, or fat tissue. In recent studies, these cells have also shown healing capability by improving angiogenesis and preventing fibrosis, which could have a role in tissue repair and tissue regeneration. Preclinical and clinical orthopedic studies conducted in the adult population support the use of MSCs for bone-healing problems, early stages of osteonecrosis, and local bone defects. Only a few published studies support the use of MSCs in pediatric osteoarticular disorders, probably due to the unknown long-term results of cellular therapy. The purpose of this review is to explain the mechanism by which MSCs could exhibit a therapeutic role in pediatric osteoarticular disorders.

$\mathbf{S}^{\mathrm{c}}$ everal pediatric osteoarticular disorders can have lifelong consequences on the patient. Scientists and physicians believe that cellular therapy with multipotent mesenchymal stem cells (MSCs) have the potential to cure these disorders, or at least significantly alleviate their symptoms and sequelae.

MSCs are adult stem cells exhibiting functional properties that have opened the way for cell-based clinical therapies. Primarily, their capacity of multilineage differentiation has been explored in a number of strategies for skeletal tissue regeneration. More recently, it has been reported that MSCs exhibit immunosuppressive and healing capacities, by improving angiogenesis and preventing apoptosis and/or fibrosis through the secretion of paracrine mediators.

Several preclinical and clinical studies support the use of MSCs in adult osteoarticular disorders such as nonunion of fractures, simple bone cyst (SBC), and osteonecrosis. However, only a limited number of case reports (1-5) have demonstrated their possible clinical applications in pediatric osteoarticular disorders.

We should identify pediatric disorders based on the availability of treatment options. In patients with no current available treatment, any promising therapy could be considered as a valid treatment. Cell-based therapy is a growing field, with many ongoing trials for the treatment of different diseases, which might be considered as a new therapeutic option specifically for some pediatric pathologies lacking any known conventional treatments.

Before adopting a specific indication of the use of MSCs in the pediatric population, it is essential that this cell-based strategy first shows adequate and promising results in animal models. However, diseases like juvenile arthritis are specific for children and distinct from adult rheumatoid arthritis, so dedicated trials are warranted for these applications. Second, all safety concerns must be evaluated for its clinical applications, and, finally, it should prove the desired results over time with minimal adverse effects for the patients.

Based on the few published reports, we believe that these conditions are not yet met, as concerns have been raised regarding the migration, biodistribution, survival, and safety of MSCs following systemic infusion or local implantation. As we mentioned earlier, if no current treatment is available or severe long-life consequences can exist, solid data of potential benefits of cell therapy far overcome the risk of their clinical use in children.

What is required at this level is a thorough review of the uses and limitations of MSCs. This will contribute to a better evaluation of the risk-benefit value and a clearer understanding of the indication of MSCs for pediatric orthopedic conditions.

\section{PROCEDURES AVAILABLE FOR MSC-BASED THERAPY}

When considering cell therapy as a new treatment option in pediatric osteoarticular disorders, it is important to analyze their potential role in local and/or systemic conditions. If a local effect is desired, an on-site injection of MSCs (with or without a scaffold) could be considered, for example, in the case of a nonunion after a long-bone fracture. On the other hand, if the intervention aims to correct a systemic disorder, two options are available. The first option would be to replace the host MSCs with unmanipulated allogeneic MSCs. This could attenuate or possibly correct genetic disorders of bone or cartilage. It is important to take into consideration that in order to allow a new population of unaltered MSCs to grow, it is necessary to create the appropriate space by partially ablating the patient's own bone marrow. This procedure is not risk-free and comes with a high potential of fatal procedures. The second

'Universidad de los Andes, Faculty of Medicine, Santiago, Chile; ${ }^{2}$ Inserm, U 844, Lapeyronie University Hospital, Montpellier, France. Correspondence: Christian Jorgensen (christian.jorgensen@inserm.fr) 
option is to use MSCs as a modulator of autoimmune chronic diseases with joints and bone clinical symptoms. The systemic interaction between MSCs and lymphocytes could result in a systemic modulation of the immune reaction. In this case, the indication of MSCs is not expected to cure but reduce the symptoms and complications.

\section{Route of Administration}

Intravascular infusion. Most of the information regarding cell therapy and osteoarticular diseases in children, especially inborn errors, comes from bone marrow transplantation (BMT), a treatment option for patients with leukemia and lymphoma. Intravascular allogeneic infusion of bone marrow for hematological disease has been successfully performed for more than $30 \mathrm{y}$, and, more recently, transplantation of umbilical cord blood stem cells has been successful (6-8).

Owen and Friedenstein first identified stem cells in the stromal tissue of bone marrow, which were capable of differentiating into different cell types, including osteogenic cells (9). In 1995, Caplan (10) identified two different stem cell lineages in the bone marrow: hematological and mesenchymal. MSCs could treat inborn bone metabolism errors by replacing the host's ill bone marrow MSCs with healthy ones from the donor. This would lead to a gradual replenishment of normal cells, an attenuation of symptoms, and eventually the cure of metabolic errors. The author theorized that osteogenesis imperfecta (OI), a genetic disease in which osteoblasts are unable to produce normal type I collagen, could be successfully treated by MSC transplantation. Horwitz et al. clinically proved this theory, publishing their results in 2001 (2).

Even though these results are promising, it is important to consider the risks involved in these interventions. The procedures are performed in "unhealthy" patients and require a partial toxic ablation of the bone marrow, which could result in complications that are life-threatening for the patient.

\section{Local injection}

Percutaneous injection. Bone marrow aspiration, without other manipulation, can be percutaneously injected at the affected site. Combining non-concentrated bone marrow with a scaffold in surgical procedures is reportedly the most used technique in clinical practice in adults patients. The use of concentrated bone marrow injection has also been described. This procedure requires centrifugation of the bone marrow, which results in a concentrated mononuclear cell sample that can be injected into the affected site (11).

By in vitro expansion, a larger number of isolated MSCs could be obtained for the treatment. These isolated and expanded MSCs can be mixed in the operation room with an osteoconductive scaffold and implanted in the affected site $(12,13)$.

Finally, in vitro expansion directly within the scaffold has also been described as an alternative method for culturing MSCs in conditions that mimic their natural environment. This requires days or weeks for cell differentiation and colonization of the scaffold. We could not find any reports of current use of this technique.
Intrarticular injection. In theory, the intrarticular injection of MSCs could be used to treat focal cartilage lesions or degenerative joint diseases. Extensive preclinical investigations support the use of MSCs in the treatment of cartilage lesions in adults; however, these results lack a direct comparison with conventional therapies such as autologous chondrocyte implantation or microfractures (14). Wakitani et al. (5), have recently published successful results, with no reported complication, of a 6-y follow-up study in four children injected intrarticularly (in the elbow) with autologous bone marrow-derived MSCs. Intrarticular injections of MSCs have been widely studied in animal models with osteoarthritis (OA); however, there are only preliminary data available for humans. Recently, an article was published that included four patients between 54 and 65 y of age, with moderate-to-severe knee OA. Each patient received an intrarticular injection of $10^{7}$ bone marrowderived autologous MSCs. The authors reported an improvement in walking time, reduction of walking pain in three of the four treated patients. No side effects were reported after a 1-y follow-up (15).

\section{Source and Characterization of MSCs}

Besides the applications discussed above, MSCs may have therapeutic value in other clinical applications by virtue of their capacity to limit scar formation through antifibrotic properties, to prevent apoptosis, to stimulate regeneration of endogenous cells, and to suppress the host immune response. Such immunosuppressive effect has been shown to occur mainly through the secretion of soluble factors by MSCs. Among the possible mediators identified, indoleamine 2,3-dioxygenase, inducible nitric oxide synthase, heme oxygenase- 1 as well as the secretion of human leukocyte antigen-G, transforming growth factor- $\beta$, interleukin-6, and prostaglandin-E2 have been postulated to play a role, but the cells need to be licensed through interferon- $\gamma$. These mechanisms result in the inhibition of the proliferation of $\mathrm{CD} 4^{+}$and $\mathrm{CD} 8^{+} \mathrm{T}$ cells, B lymphocytes, and natural killer cells; this has been demonstrated not only in vitro but also in vivo in a number of experimental models. Stem cells have been isolated from many different adult tissues including cord blood, peripheral blood, bone marrow, spleen, liver, kidney, thymus, dental pulp, periostium, skin retina, adipose tissue, and synovial tissue (16). The preference of stem cell source is determined by ease of access to tissue source, population of stem cells, and information on a particular cell system. Bone marrow-derived MSCs have been widely studied, and there is a wealth of information in the literature concerning them. They represent only $0.001-0.01 \%$ of the total nucleated cells in bone marrow aspirate (17). Bone marrow aspirate of $30 \mathrm{ml}$ only contains $\sim 1 \times 10^{5}$ cells (18). Therefore, culture and expansion in vitro are needed. MSCs can be obtained from the tissue of the patient or the donor. In case of autotransfusion, MSCs can be obtained from several sources. The most reliable and reproducible harvest processes are bone marrow aspiration and liposuction. Allogeneic MSCs can also be harvested from different tissues, including human embryonic tissue; however, due to ethical concerns, adipose and placenta have risen as the 
most used source of these cells. Adipose-derived mesenchymal cells share many properties with the MSCs harvested from bone marrow; however, collection of adipose-derived mesenchymal cells requires a less invasive procedure because the cells can be easily harvested through the liposuction of subcutaneous abdominal adipose tissue and then expanded in vitro. Even though this procedure holds an intrinsic risk of infection, adipose tissue represents an immediately available source of large of number MSCs.

\section{CLINICAL APPLICATION IN PEDIATRIC OSTEOARTICULAR DISEASES}

For this review, we propose the classification of pediatric osteoarticular disorders into two groups according to susceptibility to MSC treatment: systemic and local disorders. This relates to the administration options available for MSC therapy. This review will focus on the use of MSCs in OI, juvenile idiopathic arthritis for systemically affected children, osteonecrosis of the hip, and SBC for locally affected children.

\section{OI}

$\mathrm{OI}$ is a rare genetic disorder caused by a mutation in the COL1A1 and COL1A2 genes in chromosomes 17 and 7. This mutation results in the production of the osteoblasts of a qualitatively and quantitatively defective type I collagen (19). The prevalence of this disease is 16.3 per one million habitants (20).

OI was initially thought to be a type of rickets until 1906, when Looser first classified OI as "OI congenital" and "OI tarda." The first was characterized by multiple fractures already present at birth, and the second by multiple fractures later on. The current classifications are based on genetically specific disorders (21).

The most prevalent clinical manifestations of OI are short height, deformations of the spine and long bones, cifoescoliosis, and pectum escavatum. Other conditions such as blue sclera, hearing loss, general ligament laxity, and increased vulnerability of the skin may also be present.

Multiple fractures can occur during childbirth. The skull and ribs are soft and not completely ossified; this increases the risk of death as a result of intracranial bleeding and lung collapse.

There should be antenatal suspicion of OI if sonographically intrauterine fractures are observed. Confirmation of OI is performed through skin biopsy or DNA analysis.

A multidisciplinary approach is needed in which prevention of domestic trauma plays an elemental role. Pamidronate should also be considered, as it has shown reduction of new fractures (22). If the patient suffers a fracture, orthopedist or surgery may be needed.

In 1999, Horwitz et al. (1), despite previous reports of unsuccessful engraftment of MSCs after BMT (23), performed allogeneic BMT in three children suffering from severe OI, with standard marrow-ablative chemotherapy regimen followed by marrow infusion from either completely or partially human leukocyte antigen-matched siblings.

Two of the three patients had complete hematopoietic engraftment, and the third had mixed chimerism. Histological evidence of new healthy bone formation and clinical evidence of reduced incidence of fracture did not correlate with later osteoblast culture-expanded studies from the patients' iliac bone. No more than $2 \%$ of donor cells were found in two of the three patients, and no donor cell could be grown in the third child.

A second report by the same authors (2) showed that two of the five OI patients treated failed to exhibit engraftment of the donor MSCs, and therefore were excluded from the study. The three remaining patients showed an impressive improvement, with a reduction of bone fracture incidence, increased bone mass, and stature growth. At the 6-month follow-up, the children showed a plateau in their growth rate but a constant increase in bone mass. In their third study, Horwitz et al. (3) recruited six additional children who had received BMT and MSC transfusions, the clinical results were satisfactory, and all transplants, in contrast with the previous studies, were engrafted.

Le Blanc et al. (4) reported a case of a female fetus with multiple intrauterine fractures, genetically diagnosed with severe OI. They performed an allogeneic human leukocyte antigenmismatched male fetal MSC transfusion at the 32nd week of gestation. At 9 months of age, bone biopsy revealed the presence and persistence of $0.3 \%$ of XY-positive allogeneic cells. During the first $2 \mathrm{y}$ of life, three fractures were seen, with normal psychomotor development and growth.

Reports on allogeneic BMT have shown a partial engraftment of functional mesenchymal progenitor cells. These results could represent a feasible strategy for the treatment of OI and other forms of chondrodysplasia.

\section{Juvenile Idiopathic Arthritis}

As mentioned previously, MSCs play a role in the modulation of host immune response by inhibiting the proliferation of T lymphocytes. Therefore, the use of MSCs seems logical in diseases in which there is an increased $\mathrm{T}$-cell response such as inflammatory arthritis. Improvement in clinical and biological scores have been reported in studies using the experimental mouse with collagen-induced arthritis after the injection of MSCs derived from bone marrow or adipose tissue $(24,25)$. Nevertheless, there are contradictory results including the absence of therapeutic benefit after MSC infusion and even exacerbation of arthritis (26). Recently, our group has shown that interleukin-6-dependent prostaglandin-E2 secretion by primary murine MSCs inhibits local inflammation in experimental arthritis in a time-dependent fashion, which suggests that the discrepancy between studies may be related to the time of injection and/or the immune status of the animals at that time (27).

The mechanisms underlying the therapeutic effect needs to be further explored because no convincing increase of regulatory $\mathrm{T}$ cells has been observed in vivo (28).

Despite the immunological and clinical differences between juvenile idiopathic arthritis and rheumatoid arthritis, the autoimmune pathways seem to be similar. More clinical evidence is required to position MSCs as an effective and reliable cellular 
therapy for rheumatoid arthritis, before we can extrapolate these therapeutical effects into pediatric patients with juvenile idiopathic arthritis. Nevertheless, in patients who do not respond to the usual therapies, MSCs might be a treatment option, but no expanded recommendations can be made on the basis of current findings in the literature.

\section{SBC}

SBCs, also known as unicameral bone cysts, are fluid-filled cavities that typically appear in the long bones of growing children between the ages of 10 and $15 \mathrm{y}$. Although rare, they can also affect older children or adults. SBCs are the most common benign bone lesions, representing $3 \%$ of all primary bone lesions in children. More than $90 \%$ of SBCs are located at the proximal end of the femur and humerus (29).

The etiology of SBCs is uncertain. Several theories have been proposed to explain the pathogenesis of SBCs. Some believe that it may be due to resorptive properties of the lining or fluid of the cyst $(30,31)$, whereas others believe that it is due to venous congestion (32).

Most children with SBC are asymptomatic, and the lesion is found on unrelated routine radiographs or during consultation for local pain in the presence of a pathologic fracture. These fractures occur after low-energy traumas or even after normal sports activities. In rare cases, children may complain about local pain after weight-bearing activities (33).

The diagnosis is confirmed by a simple radiographic study. In some cases, a magnetic resonance imaging may be needed to rule out common differential diagnosis such as aneurysmatic bone cyst. The natural history of SBCs is favorable, with healing expected upon reaching skeletal maturity. Nevertheless, it is mandatory to evaluate the likelihood of a fracture without treatment in each case (34). The main objective of the treatment is to prevent pathological fractures or their recurrence.

Several treatment options have been proposed, including, open curettage and bone grafting, percutaneous injection of steroid, bone substitutes, bone marrow, calcium sulfate pellets, percutaneous decompression of the cyst, and mechanical support with internal fixation or mixture of methods to treat SBCs at multiple levels.

Open curettage and bone grafting have shown good results $(33,35)$. Scaglietti et al. was the first group to describe an improvement of healing rates after local steroid injection (36). Several studies have compared percutaneous steroid injection with percutaneous native bone marrow, but no improvements were seen after the inclusion of bone marrow (37-39). When native or concentrate bone marrow was mixed with allogeneic demineralized bone, a $56-89 \%$ healing rate was described $(40,41)$. It is important to note that according to our analysis of the available literature, steroids are the only evidence-based treatment for SBCs at the moment.

Further basic science is needed to understand the pathoetiology of SBCs and to develop future biologic solutions. Multimodal treatment strategies, such as opening the medullary canal and disrupting the cyst wall, filling the defect with a bone substitute, and possible biologic treatment of the cyst membrane, may be the best approach (42). There are no data available on SBCs treated with local expanded MSCs. However, once there is mechanical disruption of the wall, MSCs in combination with structural demineralized bone or bone substitutes could contribute to the healing process of the defect.

\section{Osteonecrosis of the Femoral Head}

Similar to other terminal vascular supply bones, such as the scaphoid and the talus, the femoral head is under the risk of suffering complications due to its impaired vascular supply.

Femoral head osteonecrosis is a condition in which there is subchondral bone death due to an insufficient vascular supply. Once the subchondral bone is affected, the cartilage loses its mechanical support and collapses.

Several risk factors have been described including traumatic lesions (e.g., displaced femoral neck fracture, hip dislocation), chronic uses of steroids, coagulopathies, and alcoholism.

Legg-Calve-Perthes disease is an idiopathic osteonecrosis of the femoral hip in children. This disease has been well defined and is the most common cause of osteonecrosis of the femoral head in children. It is usually acute and self-limited. Long-term complications are uncommon due to the high remodeling capabilities of children. Nevertheless, the risk of complications increases according to the patient's age (43). Some authors state that if it occurs in children $>12 \mathrm{y}$ old, its course is more similar to adult osteonecrosis than it is to Legg-Calve-Perthes disease. Juvenile osteonecrosis, although rare, has a higher incidence of long-term complications $(44,45)$.

Other related causes of osteonecrosis in children are hematopoietic neoplasm, chemotherapy, high doses of steroid therapies and hemoglobinopathies such as sickle cell disease. Socie et al. (46) found that 5 out of 1,264 patients at an age $<16$ y were diagnosed with osteonecrosis after receiving BMT as a treatment for different types of hematopoietic malignancies.

The factors influencing the progression of the disease from the appearance of a necrotic lesion to a subchondral fracture, femoral head collapse, and early joint replacement are not fully understood, but the size and stage of osteonecrosis have been shown to predict clinical outcomes (47-49).

Classification is mostly based on radiographic findings. The presence and extension of joint collapses are the most important risk factors for the development of OA.

Initially, patients do not feel any pain but refer to local discomfort; as time passes by, local pain can appear that usually relates to the collapse of the joint.

Current treatments aim at stopping the collapse of the joint and, therefore, preventing an early onset of $\mathrm{OA}$ in the hip.

Once the vascular supply is affected, a local inflammation process begins, and it produces edema inside the femoral head, which furthermore impairs the vascular supply. Decompression of the femoral head can be achieved by surgically removing a core of bone. This reduces intramedullary pressure and thereby prevents further vascular impingement and allows for new bone formation (50). 


\section{Review | Norambuena etal.}

This procedure has been widely accepted as a procedure for the treatment of hip osteonecrosis in early stages $(51,52)$. The clinical outcomes of core decompression had not always been satisfactory because of incomplete reconstruction of the subchondral necrotic area due to inadequate biological support (53). Biologic augmentation after core decompression has been attempted with demineralized bone matrix and bone morphogenetic protein (54). However, the relative insufficiency of osteoprogenitor cells in the proximal femur of osteonecrotic hips (55) has led to the use of bone marrow MSCs after core decompression. Several reports have shown the successful application of bone marrow MSCs through a single hole drilled into the necrotic area in patients with atraumatic osteonecrosis $(56,57)$. Recently, Gangji et al. (48) published a 5-y follow-up study in which they compared core decompression with core decompression and autologous bone marrow cell implantation in patients with pre-collapse atraumatic osteonecrosis. They found that at 60 months, 8 of the 11 hips in the control group had deteriorated to the fractural stage, whereas only 3 of the 13 hips in the bone marrow graft group had progressed to that stage. Another publication compared multiple small-drill core decompression with and without the injection of autologous concentrated bone marrow. The authors found a statistically significant improvement of clinical scores and mean hip survival outcomes in the bone marrow group at the 2-y follow-up, and they emphasized the importance of achieving $5 \times 10^{7}$ osteoprogenitor cells in the final concentration in order to effectively regenerate the necrotic area (49).

A major concern in children after core decompression is the premature halt in growth of the proximal femur due to growth plate damage.

Because Legg-Calve-Perthes typically evolves with ad integrum femoral head remodeling and children under 12 $y$ old with other causes of osteonecrosis of the femoral head achieve favorable outcomes after nonsurgical treatment; general experience with core decompression in pediatric ages is rare (58).

In an immature animal model, 12 piglets with induced femoral head osteonecrosis were treated with three smalldrill core decompressions. They found that this procedure did not produce bony physeal bars in the short term; however, multiple drilling alone did not seem to prevent femoral head deformity or promote new bone formation (59). These findings support the fact that osteoprogenitor cells are needed even in young patients. Recently, there has been a described migration of intravenous transplantation bone marrow MSCs to necrotic femoral head in an animal model (60).

To our knowledge, there is no published information on the use of a local injection of bone marrow or expanded MSCs in vitro after core decompression in children. Satisfactory results obtained after combining small-drill core decompression and concentrated autologous bone marrow MSCs in adults, in addition to the serious consequences of early-onset OA in children, will push clinical studies to confirm safety and efficacy of MSC therapy in pediatric osteonecrosis.

\section{LIMITATIONS AND FUTURE PERSPECTIVES}

Currently, there are several limitations in the clinical application of MSCs. Perhaps the most important limitation is the unknown long-term effects. Even though more than 2,000 people have been treated so far with in vitro expanded MSCs and no malignant behavior has been reported yet (61), concerns related to genetic instabilities during cellular amplification still exist (62).

Another major limitation of MSCs is engraftment capacity. In short-term follow-up, only a small percentage of MSCs are detected in the transplanted area, which creates controversy about their long-term clinical effects. Dominici et al. (63) found a lack of durable donor-derived osteopoiesis after intravenous BMT, which may reflect an intrinsic genetic program or exogenous environmental signaling that suppresses the differentiation capacity of the donor stem cells. Interesting preclinical studies have shown better engraftment results after injecting cells directly into the bone marrow cavity as compared with conventional intravenous BMT. Finally, highly standardized processes of harvest, production, and transportation of MSCs make this therapy inaccessible for some hospitals because specialized centers are required for expanding stem cells.

\section{CONCLUSION}

MSCs are no longer second-class citizens but first-line players. They show major regulatory properties in skeletal tissues controlling inflammation, immune response, and fibrosis and enhance tissue regeneration.

Promising preclinical data support the use of MSCs in adult patients; these results have led to an increasing number of studies reporting their use in clinical trials in the adult population.

As we learn more about the behavior of MSCs in adult patients, we should carefully begin to transfer the potential benefits of cell therapy to pediatric patients.

We believe that the exponential growth of knowledge in the adult population should lead to new data on the use of MSCs, which could result in treatments for pediatric diseases that currently do not have a cure.

Pediatric osteoarticular diseases have an enormous potential to be treated by MSCs. Severe inborn errors of bone metabolism, autoimmune diseases without clinical response to conventional treatment, and local bone or cartilage defects might be successfully treated by MSCs. This could be achieved by curing inborn metabolic errors, modulating autoimmune diseases, or locally regenerating bone or cartilage defects, among other benefits.

Limitations to the use of MSCs should not be considered as a major setback but rather hurdles that need to be overcome through gathering more knowledge with regard to cell therapy.

\section{STATEMENT OF FINANCIAL SUPPORT}

The authors have no relevant affiliations or financial involvement with any organization or entity with a financial interest in or financial conflict with the subject matter or materials discussed in the article. 


\section{REFERENCES}

1. Horwitz EM, Prockop DJ, Fitzpatrick LA, et al. Transplantability and therapeutic effects of bone marrow-derived mesenchymal cells in children with osteogenesis imperfecta. Nat Med 1999;5:309-13.

2. Horwitz EM, Prockop DJ, Gordon PL, et al. Clinical responses to bone marrow transplantation in children with severe osteogenesis imperfecta. Blood 2001;97:1227-31.

3. Horwitz EM, Gordon PL, Koo WK, et al. Isolated allogeneic bone marrow-derived mesenchymal cells engraft and stimulate growth in children with osteogenesis imperfecta: Implications for cell therapy of bone. Proc Natl Acad Sci USA 2002;99:8932-7.

4. Le Blanc K, Götherström C, Ringdén O, et al. Fetal mesenchymal stemcell engraftment in bone after in utero transplantation in a patient with severe osteogenesis imperfecta. Transplantation 2005;79:1607-14.

5. Wakitani S, Okabe T, Horibe S, et al. Safety of autologous bone marrowderived mesenchymal stem cell transplantation for cartilage repair in 41 patients with 45 joints followed for up to 11 years and 5 months. J Tissue Eng Regen Med 2011;5:146-50.

6. Thomas E, Storb R, Clift RA, et al. Bone-marrow transplantation (first of two parts). N Engl J Med 1975;292:832-43.

7. Thomas ED, Storb R, Clift RA, et al. Bone-marrow transplantation (second of two parts). N Engl J Med 1975;292:895-902.

8. Bertaina A, Bernardo ME, Caniglia M, Vinti L, Giorgiani G, Locatelli F. Cord blood transplantation in children with haematological malignancies. Best Pract Res Clin Haematol 2010;23:189-96.

9. Owen M, Friedenstein AJ. Stromal stem cells: marrow-derived osteogenic precursors. Ciba Found Symp 1988;136:42-60.

10. Caplan AI. Osteogenesis imperfecta, rehabilitation medicine, fundamental research and mesenchymal stem cells. Connect Tissue Res 1995;31:S9-14.

11. Hernigou P, Poignard A, Manicom O, Mathieu G, Rouard H. The use of percutaneous autologous bone marrow transplantation in nonunion and avascular necrosis of bone. J Bone Joint Surg Br 2005;87:896-902.

12. Gómez-Barrena E, Rosset P, Müller I, et al. Bone regeneration: stem cell therapies and clinical studies in orthopaedics and traumatology. J Cell Mol Med 2011;15:1266-86.

13. Buda R, Vannini F, Cavallo M, Grigolo B, Cenacchi A, Giannini S. Osteochondral lesions of the knee: a new one-step repair technique with bone-marrow-derived cells. J Bone Joint Surg Am 2010;92:Suppl 2:2-11.

14. Arøen A. Stem cell therapy for articular cartilage defects. Br Med Bull 2011;99:227-40.

15. Davatchi F, Abdollahi BS, Mohyeddin M, Shahram F, Nikbin B. Mesenchymal stem cell therapy for knee osteoarthritis. Preliminary report of four patients. Int J Rheum Dis 2011;14:211-5.

16. Khan WS, Hardingham TE. Stem Cells and Cartilage Tissue Engineering Approaches to Orthopaedic Surgery. Hauppauge, New York: Nova Science Publishers, 2010.

17. Jones EA, Kinsey SE, English A, et al. Isolation and characterization of bone marrow multipotential mesenchymal progenitor cells. Arthritis Rheum 2002;46:3349-60.

18. Bruder SP, Jaiswal N, Haynesworth SE. Growth kinetics, self-renewal, and the osteogenic potential of purified human mesenchymal stem cells during extensive subcultivation and following cryopreservation. J Cell Biochem 1997;64:278-94.

19. Shapiro JR, Sponsellor PD. Osteogenesis imperfecta: questions and answers. Curr Opin Pediatr 2009;21:709-16.

20. Wynne-Davies R, Gormley J. The prevalence of skeletal dysplasias. An estimate of their minimum frequency and the number of patients requiring orthopaedic care. J Bone Joint Surg Br 1985;67:133-7.

21. Burnei G, Vlad C, Georgescu I, Gavriliu TS, Dan D. Osteogenesis imperfecta: diagnosis and treatment. J Am Acad Orthop Surg 2008;16: 356-66.

22. Salehpour S, Tavakkoli S. Cyclic pamidronate therapy in children with osteogenesis imperfecta. J Pediatr Endocrinol Metab 2010;23:73-80.

23. Simmons PJ, Przepiorka D, Thomas ED, Torok-Storb B. Host origin of marrow stromal cells following allogeneic bone marrow transplantation. Nature 1987;328:429-32.
24. Augello A, Tasso R, Negrini SM, Cancedda R, Pennesi G. Cell therapy using allogeneic bone marrow mesenchymal stem cells prevents tissue damage in collagen-induced arthritis. Arthritis Rheum 2007;56:1175-86.

25. Gonzalez-Rey E, Gonzalez MA, Varela N, et al. Human adipose-derived mesenchymal stem cells reduce inflammatory and $\mathrm{T}$ cell responses and induce regulatory $\mathrm{T}$ cells in vitro in rheumatoid arthritis. Ann Rheum Dis 2010;69:241-8.

26. Schurgers E, Kelchtermans H, Mitera T, Geboes L, Matthys P. Discrepancy between the in vitro and in vivo effects of murine mesenchymal stem cells on T-cell proliferation and collagen-induced arthritis. Arthritis Res Ther 2010;12:R31.

27. Bouffi C, Bony C, Courties G, Jorgensen C, Noël D. IL-6-dependent PGE2 secretion by mesenchymal stem cells inhibits local inflammation in experimental arthritis. PLoS ONE 2010;5:e14247.

28. Zheng ZH, Li XY, Ding J, Jia JF, Zhu P. Allogeneic mesenchymal stem cell and mesenchymal stem cell-differentiated chondrocyte suppress the responses of type II collagen-reactive T cells in rheumatoid arthritis. Rheumatology (Oxford) 2008;47:22-30.

29. Greenspan A, Jundt G, Remagen W, Greenspan A. Differential Diagnosis in Orthopaedic Oncology, 2nd edn. Philadelphia, PA: Lippincott Williams \& Wilkins, 2007.

30. Komiya S, Minamitani K, Sasaguri Y, Hashimoto S, Morimatsu M, Inoue A. Simple bone cyst. Treatment by trepanation and studies on bone resorptive factors in cyst fluid with a theory of its pathogenesis. Clin Orthop Relat Res 1993;204-11.

31. Watanabe H, Arita S, Chigira M. Aetiology of a simple bone cyst. A case report. Int Orthop 1994;18:16-9.

32. Stanton RP, Abdel-Motaal MM. Growth arrest resulting from unicameral bone cyst. J Pediatr Orthop 1998;18:198-201.

33. Neer CS 2nd, Francis KC, Marcove RC, Terz J, Carbonara PN. Treatment of unicameral bone cyst. A follow-up study of one hundred seventy-five cases. J Bone Joint Surg Am 1966;48:731-45.

34. Makley JT, Joyce MJ. Unicameral bone cyst (simple bone cyst). Orthop Clin North Am 1989;20:407-15.

35. Spence KF Jr, Bright RW, Fitzgerald SP, Sell KW. Solitary unicameral bone cyst: treatment with freeze-dried crushed cortical-bone allograft. A review of one hundred and forty-four cases. J Bone Joint Surg Am 1976;58:636-41.

36. Scaglietti O, Marchetti PG, Bartolozzi P. The effects of methylprednisolone acetate in the treatment of bone cysts. Results of three years follow-up. J Bone Joint Surg Br 1979;61-B:200-4.

37. Wright JG, Yandow S, Donaldson S, Marley L; Simple Bone Cyst Trial Group. A randomized clinical trial comparing intralesional bone marrow and steroid injections for simple bone cysts. J Bone Joint Surg Am 2008;90:722-30

38. Chang $\mathrm{CH}$, Stanton RP, Glutting J. Unicameral bone cysts treated by injection of bone marrow or methylprednisolone. J Bone Joint Surg Br 2002;84:407-12.

39. Cho HS, Oh JH, Kim HS, Kang HG, Lee SH. Unicameral bone cysts: a comparison of injection of steroid and grafting with autologous bone marrow. J Bone Joint Surg Br 2007;89:222-6.

40. Di Bella C, Dozza B, Frisoni T, Cevolani L, Donati D. Injection of demineralized bone matrix with bone marrow concentrate improves healing in unicameral bone cyst. Clin Orthop Relat Res 2010;468:3047-55.

41. Rougraff BT, Kling TJ. Treatment of active unicameral bone cysts with percutaneous injection of demineralized bone matrix and autogenous bone marrow. J Bone Joint Surg Am 2002;84-A:921-9.

42. Donaldson S, Wright JG. Recent developments in treatment for simple bone cysts. Curr Opin Pediatr 2011;23:73-7.

43. Kim HK. Legg-Calve-Perthes disease: etiology, pathogenesis, and biology. J Pediatr Orthop 2011;31:2 Suppl:S141-6.

44. Herrera-Soto JA, Price CT. Core decompression for juvenile osteonecrosis. Orthop Clin North Am 2011;42:429-36, ix.

45. Joseph B. Natural history of early onset and late-onset Legg-Calve-Perthes disease. J Pediatr Orthop 2011;31:2 Suppl:S152-5.

46. Socié G, Cahn JY, Carmelo J, et al. Avascular necrosis of bone after allogeneic bone marrow transplantation: analysis of risk factors for 4388 patients 
by the Société Française de Greffe de Moëlle (SFGM). Br J Haematol 1997;97:865-70.

47. Karimova EJ, Rai SN, Howard SC, et al. Femoral head osteonecrosis in pediatric and young adult patients with leukemia or lymphoma. J Clin Oncol 2007;25:1525-31.

48. Gangji V, De Maertelaer V, Hauzeur JP. Autologous bone marrow cell implantation in the treatment of non-traumatic osteonecrosis of the femoral head: Five year follow-up of a prospective controlled study. Bone 2011;49:1005-9.

49. Sen RK, Tripathy SK, Aggarwal S, Marwaha N, Sharma RR, Khandelwal N. Early Results of Core Decompression and Autologous Bone Marrow Mononuclear Cells Instillation in Femoral Head Osteonecrosis A Randomized Control Study. J Arthroplasty 2011; e-pub ahead of print 12 October 2011.

50. Mont MA, Carbone JJ, Fairbank AC. Core decompression versus nonoperative management for osteonecrosis of the hip. Clin Orthop Relat Res 1996;169-78.

51. Lieberman JR. Core decompression for osteonecrosis of the hip. Clin Orthop Relat Res 2004;29-33.

52. Hungerford DS. Treatment of osteonecrosis of the femoral head: everything's new. J Arthroplasty 2007;22:4 Suppl 1:91-4.

53. Koo KH, Kim R, Ko GH, Song HR, Jeong ST, Cho SH. Preventing collapse in early osteonecrosis of the femoral head. A randomised clinical trial of core decompression. J Bone Joint Surg Br 1995;77:870-4.

54. Lieberman JR, Conduah A, Urist MR. Treatment of osteonecrosis of the femoral head with core decompression and human bone morphogenetic protein. Clin Orthop Relat Res 2004;139-45.
55. Hernigou P, Beaujean F, Lambotte JC. Decrease in the mesenchymal stemcell pool in the proximal femur in corticosteroid-induced osteonecrosis. J Bone Joint Surg Br 1999;81:349-55.

56. Hernigou P, Beaujean F. Treatment of osteonecrosis with autologous bone marrow grafting. Clin Orthop Relat Res 2002;14-23.

57. Yan ZQ, Chen YS, Li WJ, et al. Treatment of osteonecrosis of the femoral head by percutaneous decompression and autologous bone marrow mononuclear cell infusion. Chin J Traumatol 2006;9:3-7.

58. Herrera-Soto JA, Price CT. Core decompression and labral support for the treatment of juvenile osteonecrosis. J Pediatr Orthop 2011;31:2 Suppl:S212-6.

59. Gong SY, Kim HW, Park HW, Lee SY, Lee KS. Effects of multiple drilling on the ischemic capital femoral epiphysis of immature piglets. Yonsei Med J 2011;52:809-17.

60. Li ZH, Liao W, Cui XL, et al. Intravenous transplantation of allogeneic bone marrow mesenchymal stem cells and its directional migration to the necrotic femoral head. Int J Med Sci 2011;8:74-83.

61. Centeno CJ, Schultz JR, Cheever M, Robinson B, Freeman M, Marasco W. Safety and complications reporting on the re-implantation of culture-expanded mesenchymal stem cells using autologous platelet lysate technique. Curr Stem Cell Res Ther 2010;5:81-93.

62. Ross AL, Leder DE, Weiss J, Izakovic J, Grichnik JM. Genomic instability in cultured stem cells: associated risks and underlying mechanisms. Regen Med 2011;6:653-62.

63. Dominici M, Marino R, Rasini V, et al. Donor cell-derived osteopoiesis originates from a self-renewing stem cell with a limited regenerative contribution after transplantation. Blood 2008;111:4386-91. 